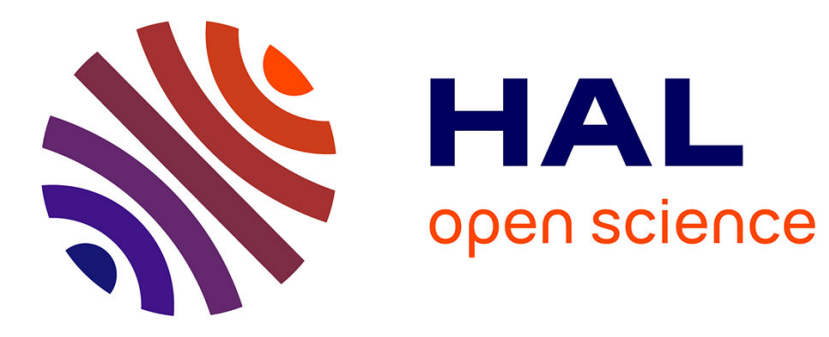

\title{
Pure dense collagen threads from extrusion to fibrillogenesis stability
}

\author{
Lise Picaut, Lea Trichet, Olivier Ronsin, Bernard Haye, Isabelle Génois, \\ Tristan Baumberger, Gervaise Mosser
}

\section{- To cite this version:}

Lise Picaut, Lea Trichet, Olivier Ronsin, Bernard Haye, Isabelle Génois, et al.. Pure dense collagen threads from extrusion to fibrillogenesis stability. Biomedical Physics \& Engineering Express, 2018, 4 (3), pp.035008. 10.1088/2057-1976/aaab78 . hal-01850246

\section{HAL Id: hal-01850246 https://hal.sorbonne-universite.fr/hal-01850246}

Submitted on 21 May 2019

HAL is a multi-disciplinary open access archive for the deposit and dissemination of scientific research documents, whether they are published or not. The documents may come from teaching and research institutions in France or abroad, or from public or private research centers.
L'archive ouverte pluridisciplinaire HAL, est destinée au dépôt et à la diffusion de documents scientifiques de niveau recherche, publiés ou non, émanant des établissements d'enseignement et de recherche français ou étrangers, des laboratoires publics ou privés. 


\title{
Pure dense collagen threads from extrusion to fibrillogenesis stability
}

\author{
Lise Picaut ${ }^{1,2}$, Léa Trichet ${ }^{2}$, Olivier Ronsin ${ }^{1}$, Bernard Haye $^{2}$, Isabelle Génois ${ }^{2}$, Tristan Baumberger ${ }^{1}$ and \\ Gervaise Mosser $^{2}$ (10) \\ 1 Institut des Nanosciences de Paris, Sorbonne Université, CNRS-UMR 7588, F-75005 Paris, France \\ 2 Sorbonne Université, Laboratoire de Chimie de la Matière Condensée de Paris, CNRS, Laboratoire de Chimie de la Matière Condensée de \\ Paris, LCMCP-UMR 7574, F-75005 Paris, France \\ E-mail: gervaise.mosser@upmc.fr
}

Keywords: collagen, extrusion process, fibrillogenesis, biomaterials, threads, tendon

Supplementary material for this article is available online

\begin{abstract}
In tendons, collagens reach a concentration of $60 \%-85 \%$ of the dry weight of the tissue, collagen I being one of the major structuring agents. Collagen is found under its fibrillated state and organized in a highly anisotropic and hierarchical manner. In a first step to synthetize a mime of a tendon, we built a set up to extrude highly viscous acidic pure molecular collagen solutions $\left(60 \mathrm{mg} \mathrm{ml}^{-1}\right)$ into meters of regular threads of $300 \mu \mathrm{m}$ to $600 \mu \mathrm{m}$ in diameter. Eight different extruding and fibrillogenesis buffers were tested for their ability to generate stable anisotropic threads, with collagen fibrils characterized by the $67 \mathrm{~nm}$-D period and optimally aligned parallel to the thread axis. Thanks to a follow up over 15 days, we pinned down one condition for which both diameter and anisotropy of the threads were stable throughout extrusion, fibrillogenesis and storage. The threads presented characteristic collagen fibrils and mechanical tests indicated a Young modulus around 1.7 MPa and an ultimate tensile strength of $0.3 \mathrm{MPa}$. Since neither chemical cross-linkers nor dehydrating processes were used, our synthesis process raises no biocompatibility or structure preservation concerns for tissue engineering applications, opening routes for further developments.
\end{abstract}

\section{Introduction}

Collagens are being used in biomaterials synthesis since they are the most abundant components of connective tissues. For instance in tendon, collagens reach a concentration of $60 \%-85 \%$ of the dry weight of the tissue [1], collagen I being the major structuring bio-component. The extracellular matrix (ECM) of tendons and ligaments is composed of many other biochemical components such as non-collagen proteins and proteoglycans $[2,1]$.

The notion of fibrillogenesis is important since, in vivo, collagen is found under its fibrillar form and more over organized in a hierarchical manner from collagen I molecules to fascicles [3]. Fibrils are characterized by a ' $67 \mathrm{~nm}-\mathrm{D}$ ' period resulting from the longitudinal arrangement of the collagen molecules within the fibrils [4]. Molecules, fibrils and fascicles are aligned in a preferred orientation in relation to the function of the tissue. For instance, excised rat-tail tendons present an overall alignment of the bundles along the tendon axis. This is clearly seen by cross-polarized optical microscopy. They also reveal units, ranging from 10 to $100 \mu \mathrm{m}$, of alternating oblique orientations to the tendon axis. The structure resulting from the repetition of those units is referred to as 'crimp' [5, 6], is a highly compliant element and was suggested to be a natural shock-absorber [7]. Elaborations of tendon-like scaffolds are inspired by this hierarchical construct and this anisotropy [8, 9]. In this project, in order to explore new paths to produce scaffolds for tendon reconstruction, we focused on the self-assembly of collagen of dense solutions following an extrusion process.

Tendons can present chronic and/or acute injuries due to intrinsic and/or external factors [10]. The etiology of tendinopathies is indeed often recognized as being multifactorial [11-13] with causes including work and sports activities, drugs [14], age and biomechanical imbalance [10]. Spontaneous regeneration is limited due to a poor vascularization and a low number of cells. Treatments can be conservative, surgical, or both, with or without rehabilitation programs 
[11, 15-17]. Surgical treatments go from sutures to insertion of metallic implants or grafts, which can be auto, xeno or allografts. Synthetic grafts made of polyester (Dacron ${ }^{\circledR}$ ), polytetrafluoroethylene (PTFE or Teflon ${ }^{\circledR}$ ) [18], silicone rubber [19], polypropylene (Marlex ${ }^{\circledR}$ mesh) [20], polyethylene teraphtalate (PET, devices Orthotape ${ }^{\circledR}$ and Orthocoupler $\left.{ }^{\circledR}\right)$ [21, 22] are also used. Depending on the system, more or less promising physiological results were obtained with, in some cases, no re-rupture occurrence observed at the level of the graft consolidation. Alongside with those synthetic grafts, grafts made out of biopolymers are also being developed to mimic as well as possible the biochemistry and three-dimensional organization of tendon in order to optimize the biocompatibility (i.e. absence of immune rejection or toxicity [23], proper host-response [24], promotion of cell adherence, proliferation and differentiation).

Another important characteristic of tendons is their mechanical properties characterized by the Young modulus and the ultimate tensile strength (UTS) as assessed under uniaxial loading along their longitudinal axis. Those vary from 0.5 to $1.9 \mathrm{GPa}$ for the Young moduli and from 50 to $120 \mathrm{MPa}$ for the UTS $[25,26]$. Such values have not yet been achieved in wet conditions with simple extruded collagen-based thread scaffolds. Drying before rehydration led to threads with Young Modulus of $46 \mathrm{MPa}$ and UTS of $10 \mathrm{MPa}$ [27-30] while the association with chemical cross-linking strategies made those values reach $766 \mathrm{MPa}$ and $110 \mathrm{MPa}$ for Young modulus and UTS respectively [27-30]. However, those treatments bring up concerns relative to biocompatibility and structure preservation. Thus new developments of scaffolds for tendon augmentation, and even more so for tendon replacement need to be undertaken to achieve scaffolds mimicking at best the tendon structural characteristics and mechanical properties to better resist solicitations and promote cell differentiation [31].

In order to address this subject, we made use of the lyotropic properties of acidic-collagen I solutions, which relate to the self-organization of collagen molecules into liquid crystal phases as soon as a critical concentration in collagen is reached [32]. These liquid crystals can be stabilized thanks to a $\mathrm{pH}$ increase to create organized fibrillar scaffolds. The $\mathrm{pH}$ increase triggers the fibrils formation while preserving the long-range liquid-crystalline organization [33]. We combined this approach with an extrusion process to generate high-density, anisotropic, collagen threads. Using a homemade extruding apparatus (figure 1), we focused on determining the best conditions to induce a stable sol-gel transition while preserving the alignment of the molecules and achieving optimal mechanical properties. For this, eight different conditions varying mainly in their ionic strengths were tested and further narrowed down to a total of three and then one condition.

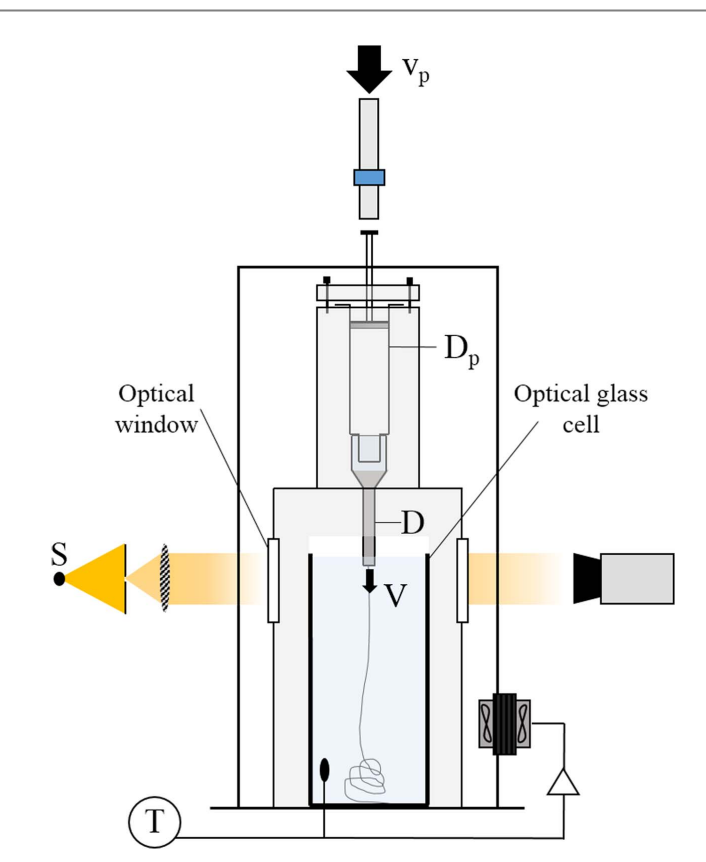

Figure 1. Extruding homemade experimental set up. A syringe $\left(\right.$ Terumo ${ }^{\circledR}$ ) of $1 \mathrm{ml}$ contains the collagen solution to be extruded. It has an inner diameter $\mathrm{D}_{\mathrm{p}}$ equal to the piston diameter of $4.45 \mathrm{~mm}$. Stainless steel blunt needles (Terumo ${ }^{\circledR}$ ) of 390 or $650 \mu \mathrm{m}$ inner diameters $\mathrm{D}_{\text {in }}$, respectively $23 \mathrm{G}$ and $20 \mathrm{G}$, are set at the syringe end. A steel rod is clamped to a stepping motorized stage, which imposes a velocity $v_{p}$ ranging from 0 to $2000 \mu \mathrm{m} \mathrm{s}^{-1}$. The average output velocity $\mathrm{v}_{\text {out }}$ is calculated as follows: $v_{\text {out }}=v_{p} \times\left(\frac{D_{p}}{D_{\text {in }}}\right)^{2}$. In order to apply the same shear condition for different inner diameters, the output velocity must be the same. The collagen solutions are extruded at $\mathrm{v}_{\text {out }}=50 \pm /-2 \mathrm{~mm} \mathrm{~s}^{-1}$. A steel structure holds the syringe with its needle as well as an optical glass cell (Hellma ${ }^{\circledR}$ ) containing the extrusion bath. Holes are drilled to create optical windows. The whole set-up is put inside a temperature-controlled enclosure. The extrusion bath can be removed at any time during the process to collect the extruded thread, while keeping the syringe in place. A quasi-parallel beam is created with a light source $(S)$ limited by a diaphragm placed at the focal length of a convergent lens. The light beam illuminates the glass cell and the extrusion flow is followed thanks to a video camera equipped with a macro-objective and placed on the other side. Acquisition speeds of 600 frames/s can be reached. This optical system allows us to obtain extrusion images of a high contrast level as shown in the figures 2(A), (B). 'T' corresponds to the thermal regulation of the extrusion chamber.

\section{Results}

Since the goal of our work was to find the best conditions to synthesize dense collagen threads that would be stable over time, present anisotropic organization of collagen fibrils and have the highest mechanical properties, we have, all along our work, narrowed down conditions, varying mainly in their ionic strengths (table 1), to determine those providing threads of optimized characteristics.

\subsection{Extrusion and maturation}

All threads look similar at the time of the extrusion in all buffers (figures 2 and SI- 1 is available online at stacks.iop.org/BPEX/4/035008/mmedia). Their surfaces present a 'sharkskin' roughness, generally 
Table 1. Fibrillogenesis buffers and collagen threads diameters ratios after 10 days of maturation. The table gives the reference number of each buffer, its composition and final ionic strength calculated as indicated in material and method section. $\mathrm{D} / \mathrm{D}_{\text {in }}$ gives an indication of the swelling of the threads in each buffer over the time of maturation. Din is the inner diameter of the extrusion needle and $\mathrm{D}$ the diameter of the thread. In this table the ratio $\mathrm{D} /$ Din is given at Day 10. Note that the $\mathrm{pH}$ was adjusted to 7.4 for all buffers.

\begin{tabular}{|c|c|c|c|c|}
\hline No & Buffers & Concentrations & Ionic strength & $\mathrm{D} / \mathrm{D}_{\text {in }}$ \\
\hline 1 & $\mathrm{Na}_{2} \mathrm{HPO}_{4}$ & $50 \mathrm{mM}$ & $150 \mathrm{mM}$ & $1.40 \pm 0.10$ \\
\hline \multirow[t]{5}{*}{2} & PBS 1X (home made) & & $165 \mathrm{mM}$ & $1.70 \pm 0.08$ \\
\hline & $\mathrm{NaCl}$ & $137 \mathrm{mM}$ & & \\
\hline & $\mathrm{KCl}$ & $2.7 \mathrm{mM}$ & & \\
\hline & $\mathrm{Na}_{2} \mathrm{HPO}_{4}$ & $8 \mathrm{mM}$ & & \\
\hline & $\mathrm{NaH}_{2} \mathrm{PO}_{4}$ & $1.5 \mathrm{mM}$ & & \\
\hline \multirow[t]{5}{*}{3} & PBS 1X (commercial) & & $166 \mathrm{mM}$ & $1.53 \pm 0.09$ \\
\hline & $\mathrm{NaCl}$ & $137 \mathrm{mM}$ & & \\
\hline & $\mathrm{KCl}$ & $2.7 \mathrm{mM}$ & & \\
\hline & $\mathrm{Na}_{2} \mathrm{HPO}_{4}$ & $8 \mathrm{mM}$ & & \\
\hline & $\mathrm{KH}_{2} \mathrm{PO}_{4}$ & $2 \mathrm{mM}$ & & \\
\hline 4 & $\mathrm{Na}_{2} \mathrm{HPO}_{4}$ & $100 \mathrm{mM}$ & $300 \mathrm{mM}$ & $1.70 \pm 0.10$ \\
\hline \multirow[t]{2}{*}{5} & $\mathrm{Na}_{2} \mathrm{HPO}_{4}$ & $50 \mathrm{mM}$ & $300 \mathrm{mM}$ & $1.43 \pm 0.06$ \\
\hline & $\mathrm{NaCl}$ & $150 \mathrm{mM}$ & & \\
\hline \multirow[t]{2}{*}{6} & $\mathrm{Na}_{2} \mathrm{HPO}_{4}$ & $50 \mathrm{mM}$ & $300 \mathrm{mM}$ & $1.32 \pm 0.05$ \\
\hline & $\mathrm{KCl}$ & $150 \mathrm{mM}$ & & \\
\hline 7 & PBS 5X (home made) & & $825 \mathrm{mM}$ & $0.98 \pm 0.05$ \\
\hline 8 & PBS 10X (home made) & & $1650 \mathrm{mM}$ & $0.82 \pm 0.04$ \\
\hline
\end{tabular}

observed when extruding viscoelastic fluids [34, 35]. The roughness amplitude is of the order of $20 \mu \mathrm{m}$ for a thread diameter of $685 \mu \mathrm{m}$ as measured on figure 2(A). Noticeably, after averaging the sharkskin roughness, the swelling ratio, measured just downstream of the die exit (the so called die-swell, of mere viscoelastic origin), remains close to 1 within the experimental error, i.e. the die swelling -an emblematic viscoelastic effect $[38,39]$ - is absent under the experimental conditions of this study. Figure 2 illustrates the threads synthesized using buffers 1, 7 and 8 as visualized during extrusion thanks to the optical system (figures 2(A) and (B)), and after the two weeks of maturation under polarized light $(C)$ and in between crossed polarizers (D). We see that the threads diameters evolve differently depending on the buffers. No changes are observed in condition 7, while swelling and shrinkage are respectively observed in conditions 1 and 8 . In figure 2(D), threads are positioned at nearly $45^{\circ}$ to both polarizers. They appear homogeneously bright along the thread axis. In the case of condition 1, the bright signal concerns only the central part of the thread. Figure 3(A) shows the evolution of the threads diameter over time during the maturation process for all buffers and figure 3(B) shows the plot of the swelling ratio after 10 days as a function of the ionic strength. The former shows that diameters evolve over 10 days and then reach a plateau. The latter reveals three groups of behaviors. For the first one, consisting in a cluster of conditions 1 to 6 , we find a $D / D_{\text {in }}>1$ indicative of a swelling of the threads. For the second one, consisting in buffers 7 , we observe a ratio $\mathrm{D} / \mathrm{D}_{\text {in }}=1$, indicating that the diameters remain constant over time. Finally for the third group, buffers
8 , we observe $D / D_{\text {in }}<1$, in agreement with the observed shrinkage of the collagen thread diameters. Data are summarized in table 1.

At this stage, we chose to focalize only on conditions 1 , as representative of the cluster of conditions 1 to 6 , as well as on conditions 7 and 8 .

\subsection{Structural characterization}

Figure 4 shows the external morphology and ultrastructures of threads obtained with fibrillogenesis buffers 1, 7 and 8 as seen by SEM. At low magnifications (200 M, $1 \mathrm{kM}, 5 \mathrm{kM}$, top three lines), we observe the overall characteristics of the threads. In condition 1 (A), threads present an outskirt of large 'hairs' while, in condition $8(\mathrm{C})$, threads present a smooth shell with hills and valleys oblique to the thread axis with some localized cracks revealing the internal ultrastructure. In condition 7 (B), the threads are smooth or with ridges running longitudinally along the thread axis with a few 'hairs' sticking here and there. At higher magnifications (bottom line), in condition 1, we see large $(\approx 560 \mathrm{~nm})$ linear fibers or bunches of compact fibrils $(\approx 150 \mathrm{~nm})$ on top of more tortuous fibrils $(\approx 70 \mathrm{~nm})$. The fibers present a large striation of $\approx 180 \mathrm{~nm}$ and sub-striations of approximatively $67 \mathrm{~nm}$. In condition 8 , we observe a smooth top shell under which linear fibers and fibrils (approximatively $150 \mathrm{~nm}$ in diameter) can be seen. The fibers present large striations of varied lengths and a sub striation of $\approx 67 \mathrm{~nm}$. Helical fibers are also clearly seen. In condition 7 , densely packed wavy fibrils with diameters of $\approx 100 \mathrm{~nm}$ are seen with the $67 \mathrm{~nm}$ cross-striation. The fibrils are fused in a more or less wavy pattern. Some larger striations can also be seen. 

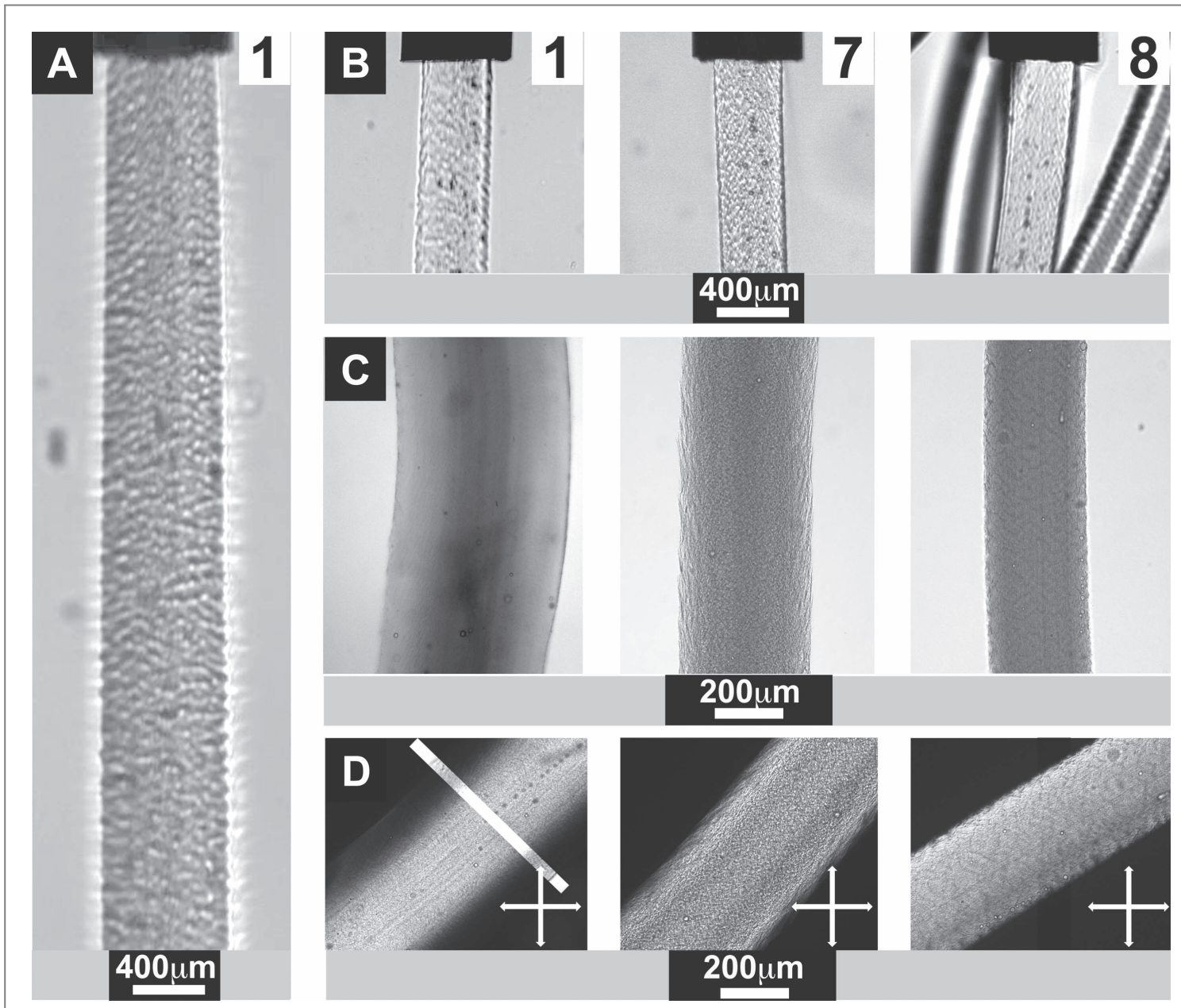

Figure $2.60 \mathrm{mg} \mathrm{ml}^{-1}$ collagen thread extrusion and maturation. Figures $2(\mathrm{~A})$ and $(\mathrm{B})$ shows the threads extrusions in extrusion buffers 1, 7 and 8 . Figure 2(A) corresponds to the extrusion through a $650 \mu$ m inner diameter needle in condition 1, while figures 2(B)-(D) correspond of extrusion in the three buffers through a $390 \mu$ m inner diameter needle. A sharkskin pattern is observed on all threads, although is less pronounced in condition 8. Figure 2(C) shows the threads after the two weeks of maturation in the respective extrusion buffers. We observe the increase and shrinkage in diameters of threads extruded in condition 1 and 8 respectively, while the diameter of the threads in condition 7 does not evolve. The thread extruded and matured in buffers 1 shows a core that is denser than the shell. Figure 2(D) shows the matured threads as seen in between cross-polarizers (crossed arrows) and at around $45^{\circ}$ from both polarizers. We see that in all the three conditions, the threads appear very bright indicating an overall alignment of the molecules. For the thread made in buffer 1, only the core shows birefringence. The shell, which limit is seen on the overlaid inset taken in bright field mode, appears dark and remains invisible. This suggests a loss of molecular orientation along the thread axis.

Figure 5 shows the distribution of the fibrils as well as their morphology as seen by transmission electron microscopy. Figures 5(A)-(C), are cross-sections of threads respectively made in buffers 1,7 and 8 , at low magnification on the top part and high magnification on the lower part. The pictures show views from the external (Ex) part to the internal part (In) of the threads as indicated by the side arrows. In $5 \mathrm{~A}$, we see that the thread presents very large fibrillar entities $(\approx 500 \mathrm{~nm}$ ) on the outmost edge of the thread. Further in, we observe large $(\approx 250 \mathrm{~nm})$ individualized fibers that do not present any specific alignment. Finally, in the core part of the thread, we observed thin fibrils $(\approx 40 \mathrm{~nm})$ tightly packed, most of them being cut perpendicular to their axis thus giving the dotted features on the photo. Figure 5(B) shows ultra structure of a thread made in buffers condition 7 . We see a cohabitation of thick $(\approx 80 \mathrm{~nm})$ fibrils together with packages or aggregates of thin fibrils ( $\approx 20 \mathrm{~nm})$. The $67 \mathrm{~nm}$
D-period is clearly seen. In figure 5(C), we observe a section of a thread made in buffers condition 8 . Here, we observe dense packages $(\approx 200 \mathrm{~nm})$ of small fibrils $(\approx 25 \mathrm{~nm})$ in majority aligned with the axis of the thread in the external part and more randomly orientated especially in the core.

\subsection{Mechanical properties}

Figure 6 shows the results of the mechanical tests and the threads after breakage. Figure 6(A) shows typical stress curves as a function of the strain obtained for the 3 selected conditions 1, 7 and 8. Figure 6(B) shows typical profile of thread extremities after breakage. figure 6(C) shows Young modulus and Ultimate Tensile Strength obtained for the three conditions as measured over more than 4 threads for each of them. We see that the highest mechanical properties are obtained for threads extruded and maturated in PBS5X that exhibit a Youngs modulus of 

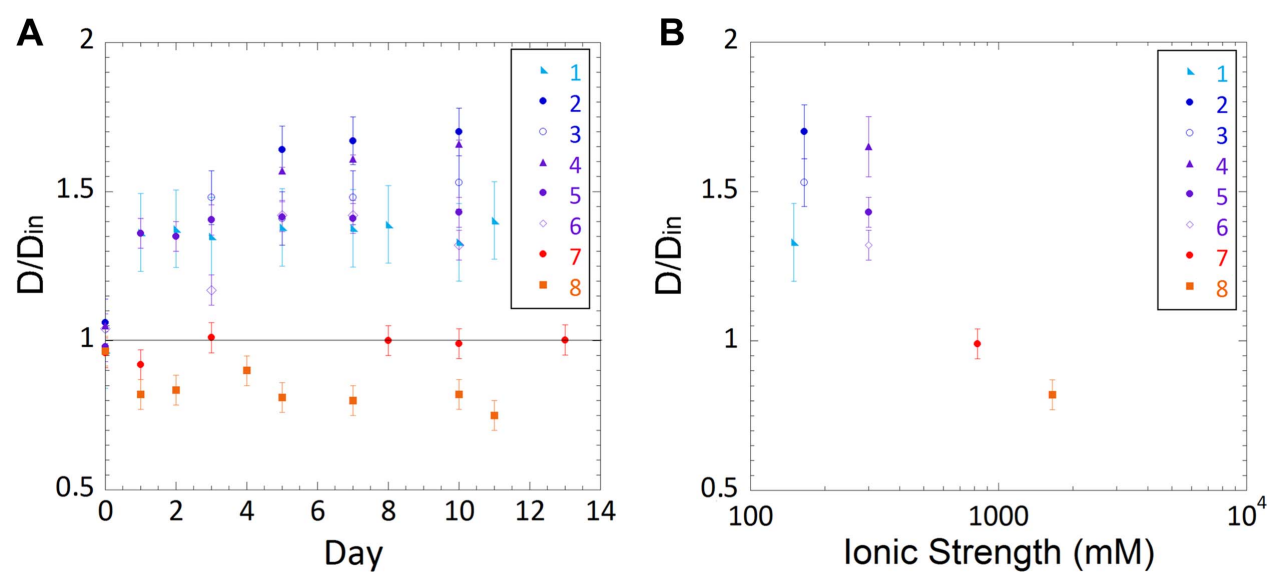

Figure 3. Evolution of thread diameters upon maturation for the different buffers. After extrusion, the threads are left in the buffers for two weeks for a maturation process. Figure 3(A) presents the evolution of the diameters of the thread, which have been measured regularly along the two weeks. We see that depending on the buffer some threads see their diameter increasing and some decreasing. In condition 7 the diameter remains relatively stable throughout the two weeks period. Figure $3(\mathrm{~B})$ shows the ratios $\mathrm{D} / \mathrm{D}_{\mathrm{in}}$, measured at Day 10, as a function of the ionic strength of the corresponding buffers. The ratios are negatively correlated to the ionic strength.

$1.66 \pm 0.45 \mathrm{MPa}$ and an ultimate tensile strength of $0.33 \pm 0.08 \mathrm{MPa}$, while threads synthetized in the two other conditions yield $0.30 \pm 0.15 \mathrm{MPa}$ and $0.13 \pm$ $0.01 \mathrm{MPa}$ respectively for Young modulus and UTS in condition 1 , and of $0.88 \pm 0.14 \mathrm{MPa}$ and $0.16 \pm$ $0.02 \mathrm{MPa}$ respectively in condition 8.

\section{Discussion}

The main objective of this work was to optimize the synthesis protocol in order to reproducibly obtain homogeneous, high-density, anisotropic collagen threads with the best mechanical properties, that is high Young modulus and high UTS. As criteria we have selected: (1) the stability of the thread upon storage in the fibrillogenesis buffers to avoid swelling or contraction, (2) the ultrastructure of the threads, (3) their mechanical properties.

Another figure of merit is the straightness of the thread. This one can be affected by several gross instabilities [34, 35]. It is controlled both by the extrusion velocity and the needle diameter. In the present work, we chose the extrusion speed according to the analysis previously made on alginate samples [36] so as to limit instabilities. We performed most of the present study with a needle of $390 \mu \mathrm{m}$ diameter to produce threads easier for post-production processes (such as weaving), as compared to collagen threads with similar properties produced in the other studies, which diameters range from 50 to $200 \mu \mathrm{m}$ [27, 29, 30, 37]. Noticeably, threads extruded at $\mathrm{D}_{\mathrm{in}}=650 \mu \mathrm{m}$ had similar structural and mechanical properties than those at $390 \mu \mathrm{m}$ diameter with adapted extrusion speed. Remarkably, upon extrusion, the thread diameter remains equal to the inner diameter of the needle. It entails that the viscoelastic iconic effect known as 'die swelling', which may result in an up to 3 -fold increase of the extrudate diameter with respect to the nominal (needle) one [38], is inhibited with our collagen solutions. This unexpected and still unexplained behavior is important since it makes it possible to control directly the thread diameter. This observation is puzzling since, even for a Newtonian fluid, a die swelling ratio of 1.1 is observed [39]. Gross slip would suppress both wall shear and normal stresses responsible for die swelling. However, it unlikely takes place in our case, since the presence of the sharkskin is both indicative of a strong adhesion of the solution at the wall and of high shear rates required to develop significant normal stresses. At this stage, one can tentatively suggest that the resulting strong anisotropy of the collagen solution could be one factor precluding the die swelling. The small irregularity of the piston drive is smoothed out by the fluidic circuit itself, which has a low pass cutoff frequency $(<1 \mathrm{~Hz})$ much smaller than the periodicity of the steps $(100-1000 \mathrm{~Hz})$. Consequently, the otherwise non-periodic sharkskin roughness cannot be attributed to the stepped nature of the drive.

Pins et al (1995) [40] have shown the importance of fibril alignment on the mechanical properties obtained, starting from a $10 \mathrm{mg} \mathrm{ml}^{-1}$ collagen thread, and using a multistep procedure with two air-dryingrehydration stages, interspersed by a stretching step of different spans to align and condense the collagen fibrils. The authors showed that on the final products, synthetized in absence of cross-linking, the linear Young modulus increased from $1,8 \mathrm{MPa}$ at $0 \%$ stretch to $46 \mathrm{MPa}$ at $50 \%$ stretch, and UTS from $0,9 \mathrm{MPa}$ to $7 \mathrm{MPa}$. They made no ultrastructural study but showed the overall increased orientation by the increase of birefringence values. They concluded that collagen fibrils alignment within the thread increased its mechanical properties. Therefore, since after extrusion our threads presented good anisotropy along their axis, it seemed important to us that the 


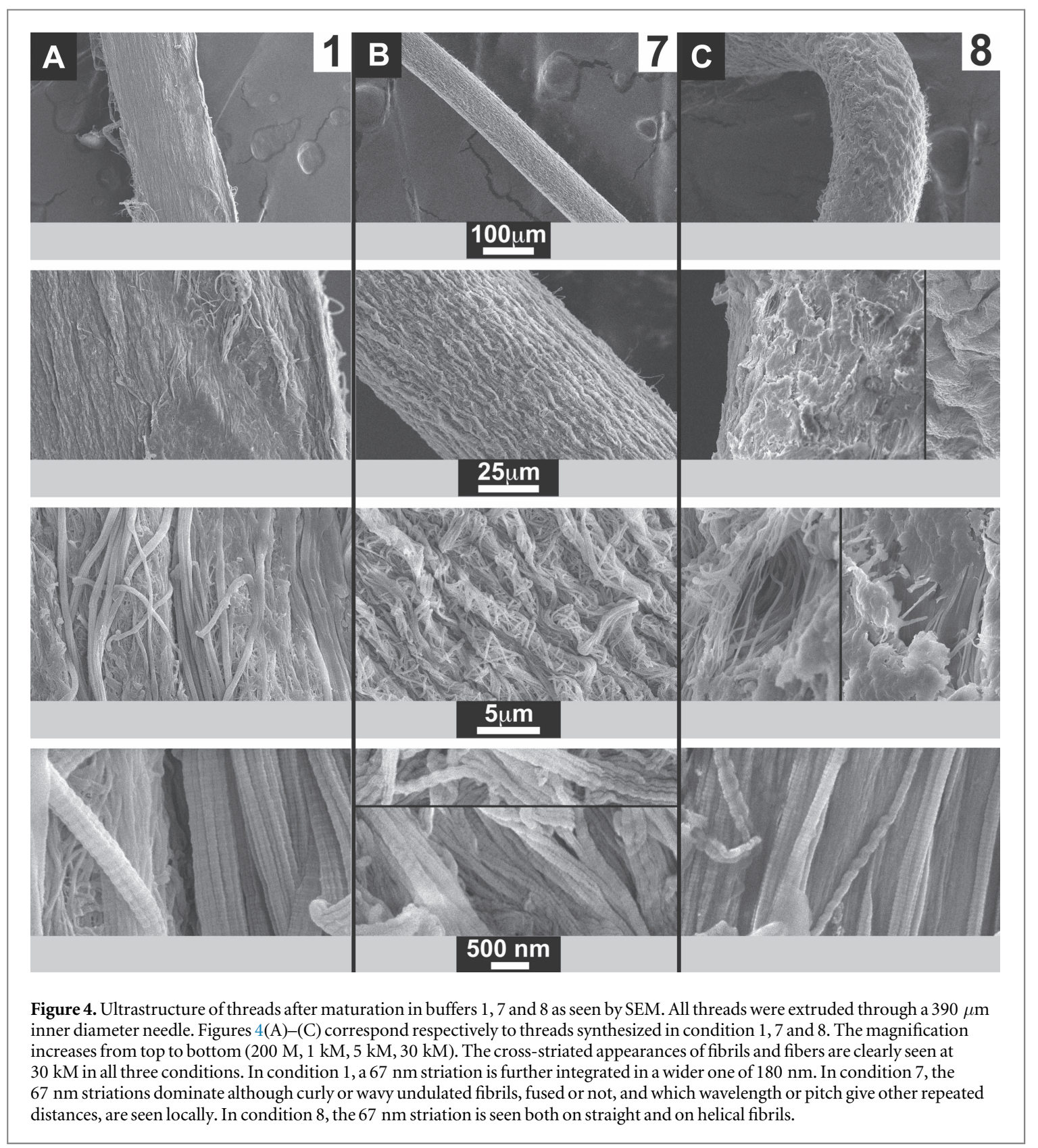

maturation and storage stages maintained this anisotropy to ensure better mechanical properties. In this context, finding conditions for which the thread diameter remained stable appeared to us mandatory as any change could potentially reflect a reorganization of the collagen molecules or fibrils, and a loss of this alignment. In our experimental conditions, after fifteen days of maturation, we see by polarized light microscopy that the overall alignment of the threads was still present for the three conditions 1, 7 and 8, but only in the central part of the thread in condition 1 . Since on the other hand our observations and measurements indicate that threads in condition 1 had swelled during the maturation process, we believe that the swelling only affected the external part, disturbing the alignment of collagen molecules and fibrils at the surface of the thread. This result points out the direct impact of the rate of fibrillogenesis upon the homogeneity of the thread. To further sustain this, we see that in the case of threads in buffers 8 , we observe shrinkage in correlation with sharkskin structure. Although this one was observed in all different buffers right after extrusion, it was only preserved in buffers 8 . In this case, the fibrillation occurred more rapidly than the fluid relaxation and faster than the osmotic swelling. It has already been shown at low collagen concentrations, that many parameters such as collagen concentration, $\mathrm{pH}$, ion types, temperature, are at play in the fibrillogenesis processes [41], and this is further confirmed at higher concentrations used in our systems. The effectiveness of phosphate anion in changing the rate of fibrillogenesis has been reported for long on diluted systems [42, 43]. Using pepsin-treated collagen $\left(4 \mathrm{mg} \mathrm{ml}^{-1}\right)$ Hayashi has shown that, at $\mathrm{pH} 6.8$, with equal concentrations of monovalent and divalent ions, the time length to reach opacity was at a 


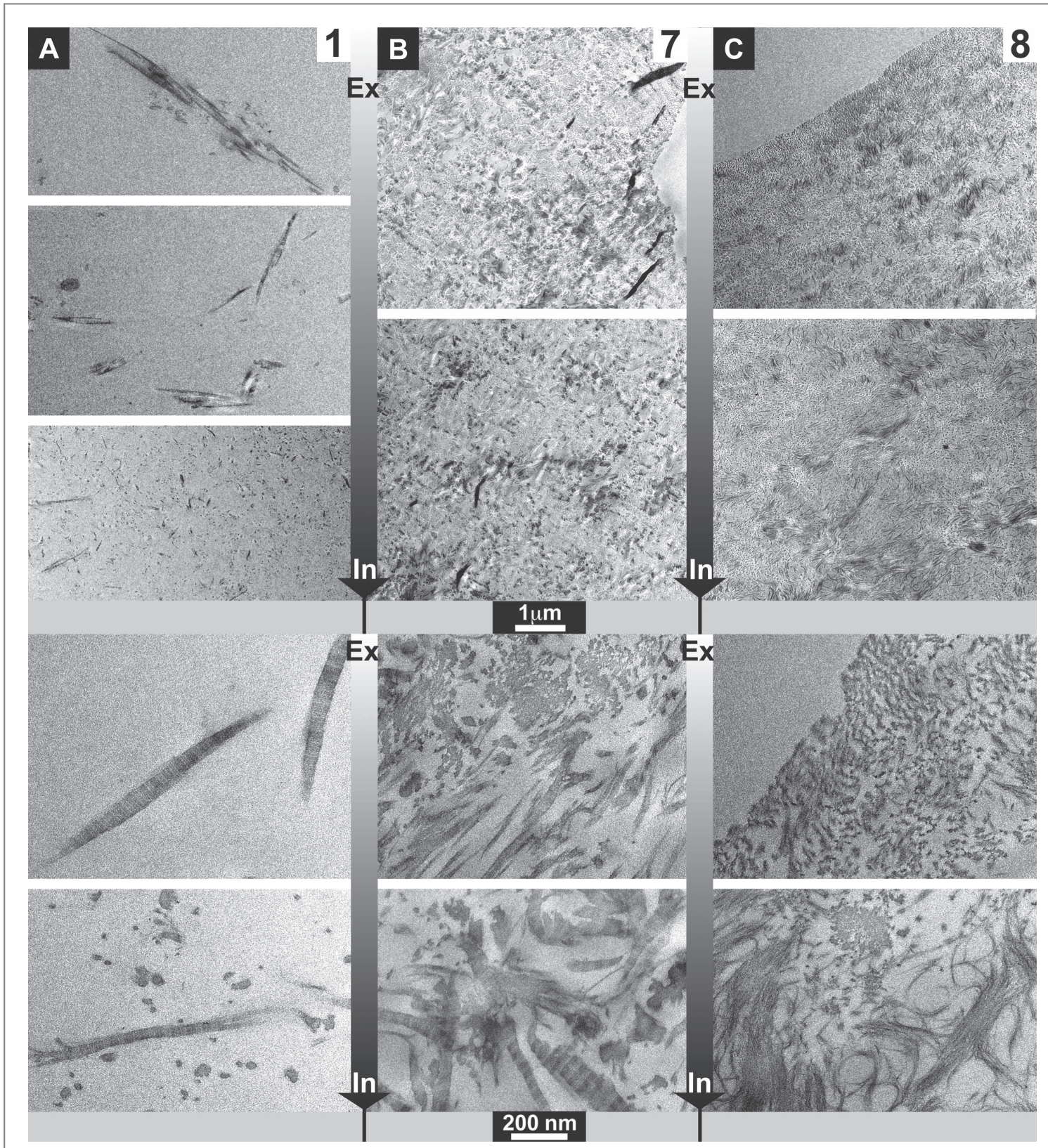

Figure 5. Ultrastructure of threads after maturation in buffers 1, 7 and 8 as seen by TEM (transverse sections). All threads were extruded through a $390 \mu$ m inner diameter needle. Figures $5(A)-(C)$ correspond respectively to threads synthesized in condition 1,7 and 8. Top: view at low magnification from the outskirt (when present) to the inner part of the thread. Bottom: view at higher magnification from the outskirt to the inner part of the thread. Collagen fibrils appear cut in all directions from longitudinal to transverse, in which case they appear as dots. In A, the thread presents three different structural characteristics when going from the outskirt of the thread to the inner part. In B and C the threads present more homogeneous appearances. In B two types of fibrils are observed, some with greater diameters than others, while in $\mathrm{C}$ most of the fibrils appear with a reduced diameter.

peak for a concentration around $150 \mathrm{mM}$ phosphate. At $\mathrm{pH} 6$, the precipitation was first retarded from 130 to $230 \mathrm{mM}$ then accelerated again at $310 \mathrm{mM}$, indicating also an effect of $\mathrm{pH}$. In our experiments, at high collagen concentration, we observed roughly that the higher the ionic strength, the faster the opacification. Our study highlights phenomena at play in dense collagen solutions, moreover constrained in cylindrical geometries, and the possible role of kinetics formation in the final structures obtained.

The ultra-structural characterization of the different threads confirmed the core-shell nature of the thread synthesized in buffers 1 . It revealed very large aggregated fibers with wide interspaces at its outmost edge, while in the internal part, smaller fibrils were found. We discarded this condition due to this pronounced difference from external to internal part. Threads made in conditions 7 and 8 both presented ultra-structures more homogeneous than in condition 1. However, in condition 8 , the threads showed a diameter contraction together with the presence of a crusty and smooth surface. This crusty and smooth surface may result from an artifact of SEM preparation although TEM images also revealed a rather continuous limit of very small fibrils. We discarded this condition since we believed the small fibril diameters, 

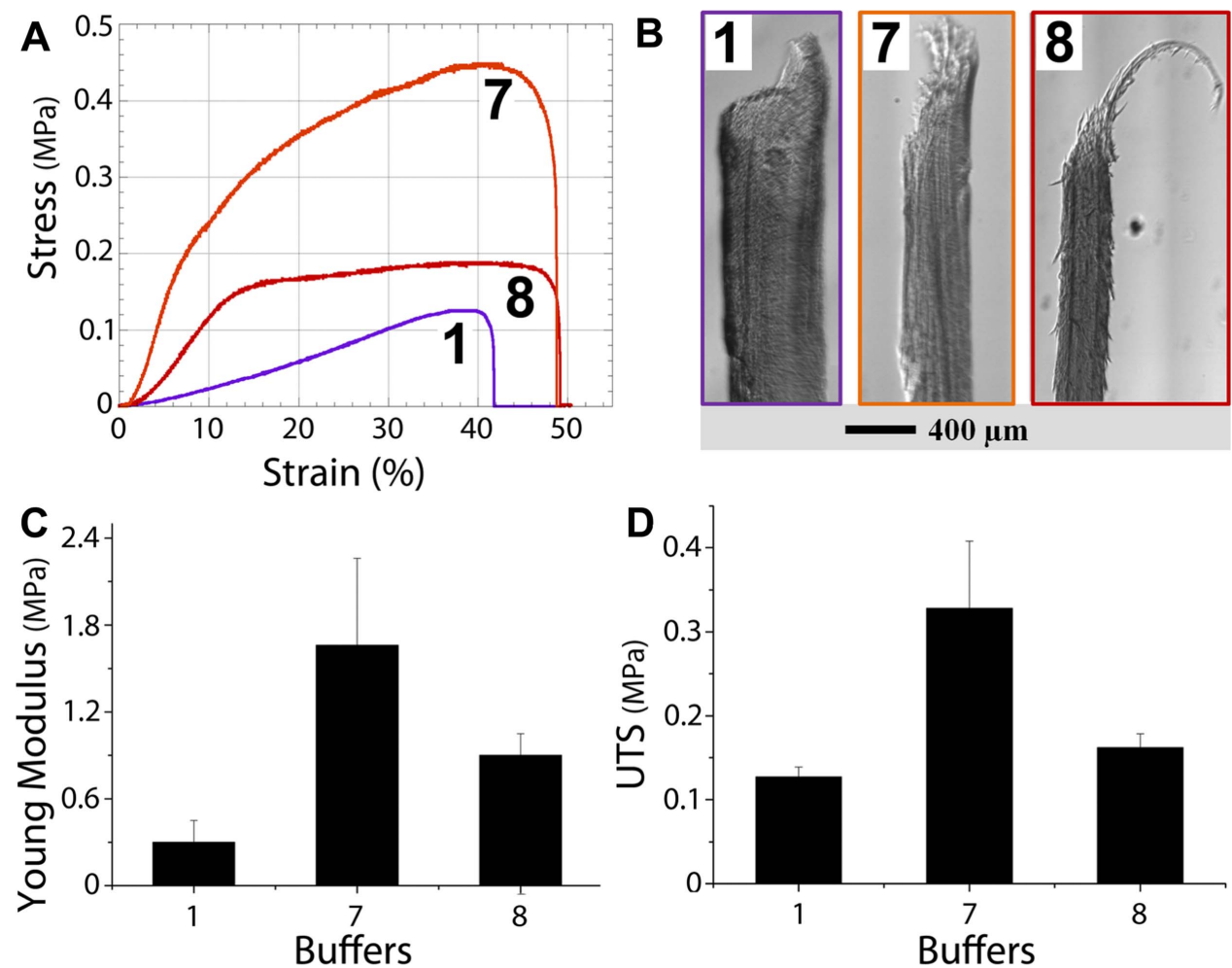

Figure 6. Mechanical characterization. All threads were extruded through a $390 \mu \mathrm{m}$ inner diameter needle. Figure 6 (A) shows the typical profiles obtained for the stress-strain curves in the three conditions 1, 7, 8. Figure 6(B) shows the three threads at the point of breakage. Note that the breakage end is rather sharp in condition 1, intermediate in condition 7 and elongated in condition 8 . Figure 6(C) gives Young Modulus and Ultimate Tensile Strength for the three conditions 1, 7 and 8 as measured over 4 threads. See Young modulus and ultimate tensile strength definitions in materials and methods (4.5).

smaller than those found in living tissues, exception made of cornea, would be unfavorable for cell attachment and colonization. Thus, although we observed two types of fibrils by TEM and SEM in the case of condition 7 , the otherwise overall homogeneity of ultra-structure seemed to us more favorable for future developments. Concerning the $67 \mathrm{~nm}$ D-bands characteristic of fibrillar collagen, although the thread diameters evolved over four days of maturation before reaching stabilization, the $\mathrm{D}$-period was still observed after two weeks in our three selected conditions (1, 7 and 8 ) indicating good and stable fibrillogenesis. This differs from the result of Uquillas et al (2011) who, on different collagen constructs and using SAXS, showed that the D-bands were present after $12 \mathrm{~h}$ incubation in PBS 1X but not in PBS 10X, and that this pattern also disappeared after $96 \mathrm{~h}$ incubation in PBS 1X [44].

Finally, concerning the mechanical properties of our threads, we see that threads made in condition 7 present the best properties. Indeed, they present the highest values of Youngs modulus $(1.66 \pm 0.45 \mathrm{MPa})$, for the highest strain at break $(48 \% \pm 5 \%)$ and the largest area under the curve that is the highest energy dissipated during the rupture process. The difference in the breaking mode of the three types of threads is also seen on their profiles adopted after rupture: in case of buffers 1, the thread breaking is sharp and localized, whereas in case of buffers 8 the breaking propagates through a sliding mode resulting in fiber-like tips at both ends. In the case of condition 7, the tip profile presents small sharp breaks observed at different positions along the longitudinal axis. From those observations, it stands out that the mechanical properties obtained in condition 7 are the best. The values obtained in this condition may seem rather low compared to other reported scaffolds [27-29, 37, 45]. However, the use of concentrated collagen solutions enables reaching moduli in the MPa range in absence of any cross-linking, airdrying and dehydration, while respecting the requirements of biocompatibility and structural preservation. Moreover, the already good mechanical properties enable further bottom-up post-processing like braiding and weaving while preserving the qualities of the original threads.

In conclusion, the homemade set up built to extrude dense and highly viscous collagen solutions enabled us to extrude regular threads over meters for collagen at $60 \mathrm{mg} \mathrm{ml}^{-1}$. Through the analysis of different buffers, we have selected a condition (PBS 5X). In this condition, the diameter of the extruded solution was preserved during fibrillogenesis and storage. Collagen fibrils, presenting the ' $67 \mathrm{~nm}-\mathrm{D}$ period', with two diameters ( 80 and $20 \mathrm{~nm}$ ) were generated, the majority of which were aligned parallel to the thread axis as revealed under cross-polarizers. However, electron microscopy reveals fibrils misaligned to this axis 
up to a $90^{\circ}$ deviation. This local disorganization, compared to the overall alignment of the fibrils within the thread, most likely interferes with the preferential sliding along the thread axis upon traction. Thus it may contribute to the mechanical strength of the threads. The threads synthesized in this condition present a Young modulus of $1.66 \pm 0.45 \mathrm{MPa}$ and an ultimate tensile strength of $0.33 \pm 0.08 \mathrm{MPa}$ without the use of any cross-linkers or any drying step. We suggest those conditions to be good candidates for future development of tendon-like scaffolds in an upgraded synthesis process enabling the generation of a crimp-like structure.

\section{Materials and methods}

\subsection{Collagen type I preparation}

Type I collagen was purified from young Wistar or Sprague-Dawley rat tail tendons as previously described [46]. Extracted tendons were washed several times with phosphate-buffered saline (PBS) to remove blood and fat traces. They were subsequently soaked and washed in $4 \mathrm{M} \mathrm{NaCl}$ solution to remove cells. Finally, tendons were extensively rinsed again with PBS and solubilized in $0.5 \mathrm{M}$ acetic acid. After successive and selective precipitations, respectively at $0.3 \mathrm{M}$ and $0.6 \mathrm{M} \mathrm{NaCl}$, the final collagen I pellets were solubilized in $500 \mathrm{mM}$ acetic acid and thoroughly dialyzed against the same solvent to completely remove salt content. The solutions were kept at $4{ }^{\circ} \mathrm{C}$ and centrifuged at $41000 \times \mathrm{g}$ (Beckman Coulter J26$\mathrm{XP})$ for $4 \mathrm{~h}$ before use. Sample purity was assessed by SDS-PAGE electrophoresis. Collagen concentration was determined from the acidic solution by titrating the amount of hydroxyproline.

Collagen solutions were concentrated using centrifugal filtration units (Vivaspin ${ }^{\circledR}$, Sartorius, with a $100 \mathrm{kD}$ cutoff) spun at $3000 \mathrm{xg}, 10^{\circ} \mathrm{C}$, until reaching the desired final concentrations $\left(60 \mathrm{mg} \mathrm{ml}^{-1}\right)$. Solutions were then placed in $1 \mathrm{ml}$ syringes and degassed by centrifugation at $3000 \mathrm{xg}$ for $30 \mathrm{~min}$ at $10^{\circ} \mathrm{C}$.

\subsection{Extrusion of dense collagen solutions}

The homemade extrusion set up, schematized in figure 1, was built to allow: (i) in-sight visualization, (ii) application of large forces compatible with highly viscous solutions, (iii) precise monitoring of the extrusion speed and the buffers exchange during extrusion. Briefly, a $1 \mathrm{ml}$ syringe, filled with a $60 \mathrm{mg} \mathrm{ml}^{-1}$ collagen solution in $500 \mathrm{mM}$ acetic acid, was mounted with a 20 or a 23-gauge (inner diameter $\mathrm{D}_{\text {in }}$ of 650 and $390 \mu \mathrm{m}$ respectively) blunt stainlesssteel needle, and loaded vertically. A motorized stage was used to bring down a rod in contact with the syringe piston and to impose its speed. The extruded fluid velocity (about $50 \mathrm{~mm} \mathrm{~s}^{-1}$ ) was adjusted so as to ensure that the system was stable against the helical instability evidenced with alginate solutions [36]. The collecting cuvette was filled with the fibrillogenesis buffer ( $\mathrm{pH}$ 7.4). At the blunt needle exit, extruded threads, plunging into the fibrillogenesis buffer, were observed and followed by an optical through-hole mounting. Prior to their use, the threads were kept and left for maturation in fibrillogenesis buffer at room temperature for 2 weeks under gentle agitation to prevent them to stick together.

Several fibrillogenesis buffers were tested with ionic strengths ranging from $0.15 \mathrm{M}$ to $1.65 \mathrm{M}$ (table 1). The buffers' $\mathrm{pH}$ were adjusted prior use to $\mathrm{pH} 7.4$ with $\mathrm{HCl}$ or $\mathrm{NaOH}$ solutions depending on the initial $\mathrm{pH}$ measured. Same buffers were used for extrusion and maturation. Ionic strength was calculated by taking into account all the ions present in the solution according to the equation $I=\frac{1}{2} \sum_{i=1}^{n} c_{i} z_{i}^{2}$, where $c_{i}$ is the molar concentration of each ion and $z_{i}$ the charge of that ion, assuming ideal solution.

\subsection{Maturation}

Collagen threads (60 $\mathrm{mg} \mathrm{ml}^{-1}$ in collagen), were kept over two weeks at room temperature in the different buffers listed in table 1 . Their diameters $\mathrm{D}$ were measured along the maturation process on at least 4 threads and on 3 different areas for each $(n>12)$. For each buffer condition and each time, standard deviation of thread diameter was determined classically as the dispersion of those threads measurements by calculating the square root of the variance.

We define the swelling ratio as $\mathrm{D} / \mathrm{D}_{\mathrm{in}}$. $\mathrm{D}$ is measured and plotted over time. Standard Deviation of this ratio was calculated by:

$$
\Delta\left(\frac{D}{\mathrm{D}_{\text {in }}}\right)=\sqrt{\left(\frac{1}{D} \times \Delta D\right)^{2}+\left(\frac{1}{D_{\text {in }}} \times \Delta \mathrm{D}_{\text {in }}\right)^{2}} \text {, where }
$$

$\Delta D$ and $\Delta \mathrm{D}_{i n}$ are the respective standard deviations of the thread diameter and the needle inner diameter measurements.

\subsection{Microscopy analysis}

For optical analysis, threads were put in a homemade thin glass-chamber filled with fibrillogenesis buffers to prevent dehydration during observation. Polarized light microscopy was performed using a transmission Nikon Eclipse E600 Pol, equipped with crossed polarizers and a Nikon DXM 1200 CCD camera.

For electron microscopy analysis, all samples were fixed using $3.63 \%$ glutaraldehyde in a cacodylate/saccharose buffer $(0.05 \mathrm{M} / 0.3 \mathrm{M}, \mathrm{pH} 7.4)$ for $1 \mathrm{~h}$ at $4{ }^{\circ} \mathrm{C}$. For scanning electron microscopy (SEM), the samples were then washed three times in a cacodylate/saccharose buffer $(0.05 \mathrm{M} / 0.3 \mathrm{M}, \mathrm{pH} 7.4)$ and dehydrated through successive ethanol baths with increasing concentrations from $70 \%$ to $100 \%$ alcohol. Thereafter, samples were dried at the carbon dioxide critical point and gold sputtered $(20 \mathrm{~nm})$ for analysis. Samples were observed at $200 \mathrm{M}, 1 \mathrm{kM}, 5 \mathrm{kM}$ and $30 \mathrm{kM}$ with a Hitachi S-3400N SEM operating at $8 \mathrm{kV}$ with a probe current around $95 \mu \mathrm{A}$. For transmission electron microscopy (TEM), samples were postfixed with $2 \%$ 
osmium tetroxide in cacodylate/saccharose buffer solution, washed in cacodylate/saccharose solution, dehydrated in successive ethanol baths (from $50 \%$ to $100 \%$ ), and finally embedded in araldite. Embedded samples were sectioned on an Ultracut Reichert Jung as already described [46]. $500 \mu \mathrm{m}$ thin sections were stained with toluidine blue and observed on a Nikon Eclipse E600 Pol microscope equipped with a $40 \times$ /0.65 NA objective and a DXM 1200 CCD camera. $70 \mathrm{~nm}$ ultrathin sections were contrasted with uranyl acetate and observed on a transmission electron microscope FEI Tecnai spirit G2 operating at $120 \mathrm{kV}$. Images were recorded on a CCD Camera (Orius Gatan 832 digital) at $4.4 \mathrm{kM}$ and $15 \mathrm{kM}$.

\subsection{Mechanical tests}

Mechanical tests were performed using a BOSE ${ }^{\circledR}$ ElectroForce ${ }^{\circledR} 3200$ Series II Test Instrument. Specimens were attached to the stainless steel crossheads by gluing each extremity with a cyanoacrylate adhesive drop. Samples and crossheads were immersed in a glass Petri dish filled with fibrillogenesis buffers at room temperature. The thread diameter $\mathrm{D}$ was determined by optical microscopy assuming full cylindrical symmetry. Mechanical loading of the thread was achieved by driving one crosshead at a constant velocity of $0.1 \mathrm{~mm}$ per second with a stepping motor (0.1-micron steps). The loading force $\mathrm{F}$ was measured with a $220 \mathrm{~g}$ load cell. We defined the nominal tensile stress as $\sigma=4 \mathrm{~F} / \pi \mathrm{D}^{2}$. The nominal strain is defined as $\varepsilon=\left(\mathrm{L}-\mathrm{L}_{0}\right) / \mathrm{L}_{0}$, where $\mathrm{L}$ is the distance between the glue points and $\mathrm{L}_{0}$ the initial length. All measurements were performed up to the specimens rupture. The resulting stress-strain curves were plotted and analyzed. At least 4 different threads were tested for each condition. The initial « toe » of the $\sigma(\varepsilon)$ curve $(\varepsilon \leq 2 \%)$ corresponds to the initial straightening of the thread. The Young modulus is determined as the slope $\mathrm{d} \sigma / \mathrm{d} \varepsilon$ in the following linear regime (typically, $2 \%<\varepsilon<5 \%$ ). The UTS is determined as the maximum stress of the $\sigma(\varepsilon)$ curve.

\section{Contributors}

Lise Picaut, did the experiments, analyzed the data and participated in writing the article.

Léa Trichet, daily supervised the work and wrote the article.

Olivier Ronsin, programmed the extrusion set up software and set the tensile testing parameters.

Bernard Haye, prepared the sample for electron microscopy observations.

Isabelle Génois, made the observations of the samples by scanning electron microscopy.

Tristan Baumberger, supervised the physical part of the work and wrote the article.

Gervaise Mosser, supervised the physico-chemical part of the work and wrote the article.

\section{Acknowledgments}

LP was supported by a PhD grant of the Ecole doctorale 397 (Physique et Chimie des Matériaux) from MESR.

\section{Conflicts of interest}

There are no conflicts of interest to declare.

\section{ORCID iDs}

Gervaise Mosser (1D https://orcid.org/0000-00029812-5340

\section{References}

[1] Screen H R C, Berk D E, Kadler K E, Ramirez F and Young M F 2015 Tendon functional extracellular matrix: TENDON FUNCTIONAL EXTRACELLULAR MATRIX J. Orthop. Res. 33 793-9

[2] Zhang G, Young B B, Ezura Y, Favata M, Soslowsky L J, Chakravarti S and Birk D E 2005 Development of tendon structure and function: regulation of collagen fibrillogenesis $J$ Musculoskelet Neuronal Interact 5 5-21

[3] Benjamin M, Kaiser E and Milz S 2008 Structure-function relationships in tendons: a review J. Anat. 212 211-28

[4] Kadler K E, Baldock C, Bella J and Boot-Handford R P 2007 Collagens at a glance J. Cell Sci. 120 1955-8

[5] Nicholls S P, Gathereole L J, Keller A and Shah J S 1983 Crimping in rat tail tendon collagen: morphology and transverse mechanical anisotropy Int. J. Biol. Macromol. 5283-8

[6] Dlugosz J, Gathercole L J and Keller A 1978 Transmission electron microscope studies and their relation to polarizing optical microscopy in rat tail tendon Micron 1969 71-82

[7] Wang J H-C 2006 Mechanobiology of tendon J. Biomech. 39 1563-82

[8] Park S A, Kim I A, Lee Y J, Shin J W, Kim C-R, Kim J K, Yang Y-I and Shin J-W 2006 Biological responses of ligament fibroblasts and gene expression profiling on micropatterned silicone substrates subjected to mechanical stimuli J. Biosci. Bioeng. 102 402-12

[9] Wang J H, Jia F, Gilbert T W and Woo S L 2003 Cell orientation determines the alignment of cell-produced collagenous matrix J. Biomech. 36 97-102

[10] Morais D S, Torres J, Guedes R M and Lopes M A 2015 Current approaches and future trends to promote tendon repair $A n n$. Biomed. Eng. 43 2025-35

[11] Thomopoulos S, Parks W C, Rifkin D B and Derwin KA 2015 Mechanisms of tendon injury and repair: TENDON INJURY AND REPAIR J. Orthop. Res. 33 832-9

[12] Young J S and Maffuli N 2007 Etiology and epidemiology of Achilles tendon problems (Achilles Tendon) ed N Maffulli and L C Almekinders (London: Springer-Verlag) 539-49

[13] Oliva F, Via A G and Maffulli N 2011 Calcific tendinopathy of the rotator cuff tendons Sports Med. Arthrosc. Rev. 19237-43

[14] Lewis T and Cook J 2014 Fluoroquinolones and tendinopathy: a guide for athletes and sports clinicians and a systematic review of the literature J. Athl. Train. 49 422-7

[15] Lomas A J et al 2015 The past, present and future in scaffoldbased tendon treatments Adv. Drug. Deliv. Rev. 84 257-77

[16] Sun C, Zhuo Q, Chai W, Chen J, Yang W, Tang P and Wang Y 2013 Conservative interventions for treating Achilles tendon ruptures Cochrane Database of Systematic Reviews The Cochrane Collaboration (Chichester, UK: John Wiley \& Sons, Ltd)

[17] Seida J C, Schouten J R, Mousavi S S, Tjosvold L, Vandermeer B, Milne A, Bond K, Hartling L, LeBlanc C and 
Sheps D M 2010 Comparative effectiveness of the nonoperative and operative treatments for rotator cuff tears Agency Healthc. Res. Qual.-Comp. Eff. Rev. 22

[18] Stapp M D 1993 Implantable materials and grafts in tendon surgery Reconstructive Surgery of the Foot and Leg: Update 93 (Tucker GA: Podiatry Institute Publishing Co) pp 289-92

[19] Williams R D and August S F 1964 Experimental evaluation of a teflon tendon prosthesis Am. J. Surg. 107913-6

[20] Choksey A, Soonawalla D and Murray J 1996 Repair of neglected Achilles tendon ruptures with Marlex mesh Injury 27 215-7

[21] Morrey M C, Barlow J D, Abdel M P and Hanssen A D 2016 Synthetic mesh augmentation of acute and subacute quadriceps tendon repair Orthopedics 39 e9-13

[22] Abdullah S 2015 Usage of synthetic tendons in tendon reconstruction BMC Proc. vol 9(BioMed Central) p A68

[23] Mertz L 2013 What is biocompatibility?: a new definition based on the latest technology IEEE Pulse 4 14-5

[24] U.S. Department of Health and Human Services Food and Drug Administration 2016 Use of International Standard ISO 10993-1, 'Biological evaluation of medical devices - Part 1: Evaluation and testing within a risk management process' (Center for Devices and Radiological Health)

[25] Ladd M R, Lee S J, Stitzel J D, Atala A and Yoo J J 2011 Coelectrospun dual scaffolding system with potential for muscle? tendon junction tissue engineering Biomater. 32 1549-59

[26] Jung H-J, Fisher M B and Woo S L-Y 2009 Role of biomechanics in the understanding of normal, injured, and healing ligaments and tendons BMC Sports Sci. Med. Rehabil. 1 $1-17$

[27] Cornwell K G, Lei P, Andreadis S T and Pins G D 2007 Crosslinking of discrete self-assembled collagen threads: effects on mechanical strength and cell-matrix interactions J. Biomed. Mater. Res. A 80A 362-71

[28] Pins G D, Huang E K, Christiansen D L and Silver F H 1997 Effect of static axial strain on the tensile properties and failure mechanisms of self-assembled collagen fibers J. Appl. Polym. Sci. 63 1429-40

[29] Dunn M G, Avasarala P N and Zawadsky J P 1993 Optimization of extruded collagen fibers for ACL reconstruction J. Biomed. Mater. Res. 27 1545-52

[30] Kato Y P and Silver F H 1990 Formation of continuous collagen fibres: evaluation of biocompatibility and mechanical properties Biomater. 11 169-75

[31] Tong W Y et al 2012 Functional replication of the tendon tissue microenvironment by a bioimprinted substrate and the support of tenocytic differentiation of mesenchymal stem cells Biomater. 33 7686-98
[32] Giraud-Guille M-M, Besseau L and Martin R 2003 Liquid crystalline assemblies of collagen in bone and in vitro systems J. Biomech. 36 1571-9

[33] Gobeaux F, Mosser G, Anglo A, Panine P, Davidson P, Giraud-Guille M-M and Belamie E 2008 Fibrillogenesis in dense collagen solutions: a physicochemical study J. Mol. Biol. 376 1509-22

[34] Larson R G 1992 Instabilities in viscoelastic flows Rheol. Acta 31 213-63

[35] Denn M M 2001 Extrusion instabilities and wall slip Annu. Rev. Fluid Mech. 33 265-87

[36] Picaut L, Ronsin O, Caroli C and Baumberger T 2017 Experimental evidence of a helical, supercritical instability in pipe flow of shear thinning fluids Phys. Rev. Fluids 2 1-17

[37] Cavallaro J F, Kemp P D and Kraus K H 1994 Collagen fabrics as biomaterials Biotechnol. Bioeng. 43 781-91

[38] Miller J C 1963 Swelling behavior in extrusion Polym. Eng. Sci. 3 134-7

[39] Tanner R I 1970 A theory of die-swell J. Polym. Sci. Part B Polym. Phys. 8 2067-78

[40] Pins G D, Huang E K, Christiansen D L and Silver F H 1997 Effect of static axial strain on the tensile properties and failure mechanisms of self-assembled collagen fibers J. Appl. Polym. Sci. 63 1429-40

[41] Mertz E L and Leikin S 2004 Interactions of inorganic phosphate and sulfate anions with collagen Biochemistry (Mosc.) 43 14901-12

[42] Hayashi T and Nagai Y 1973 Factors affecting the interaction of collagen molecules as observed by in vitro fibril formation J. Biochem. (Tokyo) 74 253-62

[43] Williams B R, Gelman R A, Poppke D C and Piez K A 1978 Collagen fibril formation. Optimal in vitro conditions and preliminary kinetic results J. Biol. Chem. 253 6578-85

[44] Uquillas J A, Kishore V and Akkus O 2011 Effects of phosphate-buffered saline concentration and incubation time on the mechanical and structural properties of electrochemically aligned collagen threads Biomed. Mater. 6 035008

[45] Kato Y P, Christiansen D L, Hahn R A, Shieh S J, Goldstein J D and Silver F H 1989 Mechanical properties of collagen fibres: a comparison of reconstituted and rat tail tendon fibres Biomater. $1038-42$

[46] Tidu A, Ghoubay-Benallaoua D, Lynch B, Haye B, Illoul C, Allain J-M, Borderie V M and Mosser G 2015 Development of human corneal epithelium on organized fibrillated transparent collagen matrices synthesized at high concentration Acta Biomater. 22 50-8 